\title{
Avanços metodológicos na avaliação de alimentos e de exigências nutricionais para aves e suínos
}

\author{
Horacio S. Rostagno', Silvano Bünzen², Nilva K. Sakomura ${ }^{3}$ e Luiz F.T. Albino ${ }^{1}$ \\ 1 - Professor Titular, Departamento de Zootecnia. Universidade Federal de Viçosa. Viçosa, MG, Brasil. 36571-000. \\ 2 - Estudante de Doutorado em Zootecnia, UFV. \\ 3 - Professora Titular, UNESP. Jaboticabal, SP.
}

RESUMO - A produção industrial de aves e suínos mostrou enormes avanços nos últimos anos, principalmente devido ao aumento dos conhecimentos na área de genética e de nutrição. Na área de avaliação dos alimentos e das exigências nutricionais de animais monogástricos, as melhoras se dão a passos largos, devido á seriedade com que os profissionais encaram a responsabilidade de fazer pesquisa de qualidade dentro e fora do país. Neste trabalho serão abordadas as metodologias que permitem melhorar a utilização dos alimentos de maneira mais eficiente e econômica. Serão citados alguns cuidados e procedimentos essenciais para executar adequadamente experimentos de desempenho com aves e suínos. Na atualidade, para a realização de experimentos com monogástricos, é necessário: definir claramente os objetivos, utilizar animais com peso inicial uniforme, usar número adequado de repetições e de animais por unidade experimental. Testes de médias devem ser usados para as variáveis qualitativas e quando a variável independente for quantitativa aplicar análise de regressão. O nível de significância utilizado $(5,7,10 \%)$ pode variar conforme a importância econômica da característica estudada. A adequada condução dos ensaios, sejam de crescimento ou de digestibilidade, é fundamental para que as ferramentas apresentadas possam ter efetividade, resultando na melhora da produtividade e na redução dos custos e da excreção de nutrientes.

Palavras-chaves: alimentos, exigências, metodologias, monogástricos

\section{Methodological improvements in feedstuffs evaluation and nutritional requirements for poultry and swine}

\begin{abstract}
Swine and Poultry production showed enormous progress in the last few years, mainly due to the increased knowledge in genetics and nutrition. In the area of feedstuffs evaluation and nutritional requirements the improvement also was big due to the seriousness of the professionals to make high quality research in Brazil and abroad. This paper shows methodologies that can improve feedstuffs utilization more efficiently with lower costs. Procedures are described for adequate execution of growth experiments with poultry and swine. Actually, to run experiments with monogastric animals it is important to: define clearly the objectives, utilize animals with uniform starting weight, to use adequate number of replicates and animals per experimental unit. Mean test comparation should be used for qualitative variable and regression analysis when the independent variable is quantitative. The level of significance applied may vary $(5,7,10 \%)$ depending upon the economic importance of the parameter evaluated. Adequate execution of growth or digestibility trials is of fundamental importance for the recommendations made in this paper to be effective and result in increased productivity and reduction of costs and nutrient excretion.
\end{abstract}

Key words: feedstuffs, methodologies, monogastrics, requirements

\section{Introdução}

A produção industrial de aves e suínos sofreu enormes avanços nos últimos anos, assumindo caráter de importância fundamental para a economia de nosso país. Grande parte deste crescimento está Correspondências devem ser enviadas para: rostagno@ufv.br associada ao conhecimento do valor nutricional dos ingredientes das rações e das exigências nutricionais dos animais nas diferentes fases produtivas, bem como em melhorias de manejo e ambiência.

O objetivo principal é o aumento da produtividade de aves e suínos, via melhora do desem- 
penho utilizando mais eficientemente nas rações, alimentos tradicionais e alternativos. Simultaneamente, as exigências nutricionais dos animais deveriam ser adequadamente ajustadas para evitar o excesso de nutrientes nas dietas que são eliminados nas fezes e urina, podendo contaminar o solo e as reservas de água.

Dessa forma, é preciso que as pesquisas na área da nutrição animal levem em consideração fatores que possam interferir nos resultados dos experimentos, incluindo genética animal, ambiente e manejo, de modo a aumentar a confiabilidade dos resultados, e buscando sempre avançar no conhecimento das metodologias usadas na avaliação dos alimentos e na determinação das exigências nutricionais de aves e suínos.

\section{Composição e valor nutritivo dos alimentos}

\section{Conteúdo de aminoácidos}

Na elaboração de rações para animais monogástricos é de fundamental importância o conhecimento do valor nutricional dos alimentos, representado pelo conteúdo de aminoácidos, coeficientes de digestibilidade dos nutrientes e valores energéticos. Estes valores possibilitam a elaboração de rações otimizando o aproveitamento dos nutrientes pelos animais, evitando deficiências ou excesso de nutrientes, o que tanto auxilia na diminuição de custos, quanto na excreção de nutrientes no ambiente.

O conteúdo de aminoácidos dos alimentos pode ser determinado mediante o analisador de aminoácidos, metodologia demorada e de alto custo. $\mathrm{Na}$ atualidade, opção mais viável utilizada por várias empresas é o equipamento NIRS (Near Infra Red Spectrometry), ou seja, aparelho de Refletância no Infravermelho Próximo. O funcionamento do NIRS baseia-se na absorção de energia monocromática infravermelha, por ligações químicas dos grupos funcionais dos nutrientes. $\mathrm{O}$ espectro obtido é relacionado com uma amostragem padrão, mediante a utilização de equações de predição préestabelecidas. São necessárias no mínimo 60 amostras de cada alimento para elaborar as equações de calibração do aparelho.

O uso do NIRS para estimar o conteúdo de aminoácido total dos alimentos é uma ferramenta importante para a indústria de rações, pois é rápida e

๑ 2007 Sociedade Brasileira de Zootecnia de baixo custo, tornando a formulação mais precisa que em termos de conteúdo de aminoácido total. Entretanto, a introdução de maior número de variáveis (digestas) proporciona maior variação, sendo necessário descartar aproximadamente $10 \%$ das amostras avaliadas, cujos espectros estejam considerados fora do padrão (Jackson \& Dalibard, 1995).

\section{Digestibilidade dos nutrientes - aminoácidos}

Os valores de nutrientes digestíveis são normalmente encontrados nas tabelas de composição de alimentos (NRC, CVB, Rostagno et al., 2005). No entanto, devido á variação das condições experimentais (idade dos animais, genótipo, nível de alimentação) muitas destas tabelas apresentam informações distintas, o que sugere a necessidade de utilizar valores adequados ás condições brasileiras para permitir a expressão do potencial máximo de crescimento dos animais (Abreu et al., 2004).

O valor nutritivo da proteína de um alimento depende de sua composição em aminoácidos, de sua digestibilidade e disponibilidade. Os ensaios para estimar a disponibilidade de aminoácidos são baseados em ensaios de crescimento e, embora determinem valores mais precisos, uma vez que representam a porção que realmente esta sendo utilizada pelos animais, apresenta limitações quanto ao uso, exigindo ensaios com duração maior de tempo e fornecem ainda informações de apenas um aminoácido por ensaio. Este é o principal motivo que torna os valores de aminoácidos digestíveis mais utilizados.

A digestibilidade dos aminoácidos, por sua vez, procura avaliar a diferença entre a quantidade dos aminoácidos ingeridos e os que são excretados. No entanto, por haver influência (síntese ou destruição de aminoácidos) dos microorganismos presentes no intestino grosso do animal, a melhor opção foi de estimar a digestibilidade dos aminoácidos na porção terminal do íleo, cujo conteúdo ainda não sofreu interferência da flora intestinal. Os valores são expressos em digestibilidade ileal verdadeira dos aminoácidos, com a correção pelas perdas endógenas de aminoácidos, provenientes das enzimas, mucinas e células intestinais de descamação.

Várias metodologias são usadas para a obtenção dos coeficientes de digestibilidade 
verdadeira dos aminoácidos. Para aves, normalmente se utilizam frangos em crescimento com a metodologia do sacrifício e coleta do conteúdo de digesta na porção terminal do ileo. Outra opção é a utilização de galos adultos cecectomizados (Pupa et al., 1998). Em suínos, utilizam-se animais canulados na porção final do íleo, ou a técnica da anastomose íleoretal, evitandose assim, a influência dos microorganismos presentes no intestino grosso. Na Tabela 1 são mostrados os valores de aminoácidos digestíveis verdadeiros determinados com aves e suínos.

Vários experimentos mostraram que as rações calculadas usando dados de aminoácidos digestíveis verdadeiros resultam em melhor desempenho dos animais e maiores benefícios econômicos (Rostagno et al., 1995).

\section{Digestibilidade dos nutrientes - fósforo}

O mineral fósforo, além de ser o terceiro nutriente mais oneroso nas rações para monogástricos, atrás de proteína e energia, tem sido considerado um dos nutrientes mais poluidores. Tal fato reside em grande parte pelo baixo aproveitamento do fósforo das fontes vegetais pelas aves e suínos, exigindo a suplementação de fontes inorgânicas de fósforo, o que leva a formulação de dietas com alto conteúdo de fósforo total e, conseqüentemente, altas excreções nos dejetos.

Várias pesquisas têm sido conduzidas a fim de determinar a digestibilidade e/ou disponibilidade do fósforo nos diversos alimentos utilizados nas rações de aves e suínos. Jongbloed et al. (1992) cita que a digestibilidade é uma metodologia pratica e rápida para determinar a digestibilidade do fósforo nos alimentos, através da condução de ensaios em gaiolas metabólicas, uma vez que, para avaliar a disponibilidade deste mineral utilizam-se ensaios de crescimento que demandam maior tempo e número de animais, além do sacrifício destes para avaliar a deposição do mineral nos tecidos (ossos).

Os ensaios para determinação de digestibilidade do fósforo nos alimentos são conduzidos em gaiolas metabólicas, de forma semelhante à determinação dos valores energéticos dos alimentos, realizando a coleta de fezes ou de digesta, para análise da quantidade fósforo excretado. Posteriormente, mediante a determinação do fósforo ingerido menos o excretado, determinase a quantidade absorvida de cada alimento. $\mathrm{Na}$ Tabela 2 são mostrados os coeficientes de digestibilidade do fósforo, de alguns alimentos, determinados com aves e suínos.

\section{Valor energético dos alimentos}

O valor energético dos alimentos, estimado via ensaios de digestibilidade, utilizando gaiolas de metabolismo (no caso de suínos) ou em baterias metálicas (aves) onde, após o fornecimento das dietas experimentais são coletadas as fezes e urina e/ ou excretas. As dietas e o material coletado são analisados em bomba calorimétrica, fornecendo o valor de energia bruta (EB). A segunda etapa

Tabela 1 - Conteúdo e coeficiente de digestibilidade ileal verdadeira dos aminoácidos de alguns alimentos para aves e suínos (matéria natural, \%).

\begin{tabular}{lccccccc}
\hline & \multicolumn{3}{c}{ Lisina } & & \multicolumn{3}{c}{ Treonina } \\
\cline { 2 - 3 } \cline { 6 - 7 } & Total & DigestívelAves & DigestívelSuínos & & Total & DigestívelAves & DigestívelSuínos \\
\hline Milho & 0,24 & $0,21(85,9)$ & $0,19(79,8)$ & & 0,32 & $0,27(83,8)$ & $0,26(81,0)$ \\
Sorgo & 0,20 & $0,17(84,8)$ & $0,16(79,4)$ & & 0,31 & $0,26(85,4)$ & $0,25(83,6)$ \\
Flo de Soja & 2,77 & $2,55(92,2)$ & $2,53(91,3)$ & & 1,78 & $1,57(88,6)$ & $1,55(87,3)$ \\
Flo de trigo & 0,62 & $0,47(75,5)$ & $0,46(74,6)$ & & 0,51 & $0,37(72,1)$ & $0,37(71,4)$ \\
\hline
\end{tabular}

Adaptado de Rostagno et al., 2005.

Tabela 2 - Coeficientes de digestibilidade do fósforo em alimentos para aves e suínos (matéria natural, \%).

\begin{tabular}{lcccc}
\hline & $\begin{array}{c}\text { Conteúdo } \\
\text { de P total }\end{array}$ & \multicolumn{2}{c}{$\begin{array}{c}\text { Coeficiente de Digestibilidade } \\
\text { Suínos }\end{array}$} & $\begin{array}{c}\text { Coeficiente de Digestibilidade } \\
\text { Aparente - Aves }^{\mathbf{1}}\end{array}$ \\
\cline { 3 - 4 } & & Aparente $^{\mathbf{1}}$ & Verdadeiro $^{\mathbf{2}}$ & \\
\hline Milho & 0,24 & 20 & 68 & 30 \\
Farelo de Soja & 0,59 & 39 & 52 & 42 \\
Farelo de Trigo & 0,94 & 30 & 56 & 37 \\
Fosfato Bicálcico & 18,50 & 70 & 66 & 90 \\
\hline
\end{tabular}

1 - Adaptado de CVB, 1998. 2 - Adaptado de Bünzen et al., 2006. 
consiste em calcular os valores de energia digestível (ED) do alimento, subtraindo o valor de EB encontrada nas fezes. Os valores de energia metabolizável (EM) são obtidos pela diferença entre a $\mathrm{ED}$ e as perdas de energia na urina.

$\mathrm{O}$ conhecimento das exigências nutricionais e do aproveitamento dos nutrientes pelos animais tem evoluído para uma tendência em usar valores de energia líquida dos alimentos, através de equações propostas inicialmente por Noblet $e t a l$. (1994), porque parte da EM se perde nos trabalhos de digestão, absorção e metabolismo dos nutrientes na forma de incremento calórico (IC). A energia líquida é calculada por modelos matemáticos que estimam o gasto energético do incremento calórico (EM - IC) da proteína, carboidratos e gorduras.

Tendências atuais têm proposto, no caso de suínos, a diferenciação dos valores de energia líquida para suínos em crescimento e porcas adultas (gestantes e lactantes), principalmente para alimentos fibrosos, uma vez que, por possuírem maior tamanho do trato gastrointestinal, ocorre maior fermentação dos microorganismos no intestino grosso, produzindo ácidos graxos voláteis que são aproveitados como fonte de energia pelos suínos (Noblet et al., 2004).

As análises dos laboratórios de controle de qualidade das indústrias são pouco usadas para corrigir o valor nutritivo dos ingredientes. Para a indústria de rações o uso de equações é de extrema importância, não somente para determinar o valor energético dos alimentos, mas também para realizar os ajustes necessários de acordo com a variação da composição, principalmente de proteína, gordura e fibra dos ingredientes. $\mathrm{O}$ uso de equação de predição da energia permite maximizar a utilização dos dados de composição obtidos mediantes análises laboratoriais de rotina.
Rostagno et al. (2005) publicaram equações para estimar os valores energéticos dos alimentos, para aves e suínos, que podem ser usadas para corrigir e ajustar as matrizes de energia pelos nutricionistas da indústria de rações (Tabela 3). Para facilitar o uso das equações foi desenvolvido o programa Calculador, em planilhas formato Excell, encontrado no CD que acompanha as Tabelas Brasileiras, bastando introduzir os dados de composição que a energia do alimento, para aves e suínos, é calculada.

Exemplos do uso das equações usando o programa Calculador são mostrados na Tabela 4 , onde a redução do conteúdo de $1 \%$ da proteína e da gordura do milho, comparado aos valores calculados das Tabelas Brasileiras, resultou no decréscimo de 123 e $122 \mathrm{kcal}$ de EM/kg para aves e suínos, respectivamente. Entretanto o aumento de $2 \%$ da proteína e da gordura da farinha de carne e ossos mostrou valores de 168 e $159 \mathrm{kcal}$ de EM/ kg superiores para aves e suínos, respectivamente. Os ajustes dos valores energéticos das rações, de acordo com a composição dos ingredientes, resultarão em desempenhos mais facilmente previsíveis

\section{Experimentos de desempenho}

\section{Distribuição dos Animais}

Pesquisas envolvendo ensaios de produção com animais para avaliar características de desempenho exigem cuidados fundamentais, como o planejamento criterioso do experimento, definição dos objetivos (hipóteses a serem testadas e/ou efeitos a serem estimados), revisão de literatura que permita a obtenção de conhecimentos prévios relacionados ao assunto, escolha das variáveis avaliadas, aplicação correta de técnicas estatísticas e interpretação dos resultados.

Tabela 3 - Equações para estimar os valores de energia dos alimentos para aves e suínos

\begin{tabular}{|c|c|}
\hline Aves & \\
\hline Alimentos de origem vegetal & $\mathrm{EMn}=4,31 \mathrm{PBd}+9,29 \mathrm{Gd}+4,14 \mathrm{ENNd}$ \\
\hline Alimentos de orig, animal e gorduras & $\mathrm{EMn}=4,31 \mathrm{PBd}+9,29 \mathrm{Gd}$ \\
\hline Suínos & \\
\hline Alimentos de origem vegetal & $\mathrm{EM}=4,952 \mathrm{PBd}+9,45 \mathrm{Gd}+4,14(\mathrm{MOd}-\mathrm{PBd}-\mathrm{Gd})$ \\
\hline Alimentos de origem animal & $\mathrm{EM}=4,952 \mathrm{PBd}+9,45 \mathrm{Gd}$ \\
\hline Gorduras e Carboidratos & $\mathrm{EM}=0,965 \mathrm{ED}$ \\
\hline$E L=0,73 \mathrm{I}$ & $3,1 \mathrm{G}+3,7 \mathrm{~A}-6,7 \mathrm{~PB}-9,7 \mathrm{FB}$ \\
\hline EM = Energia metabolizável, kcal/kg & EL= Energia Líquida, kcal/kg \\
\hline $\begin{array}{l}\mathrm{PBd}=\text { Protéína Digestível, } \mathrm{g} / \mathrm{kg} \\
\mathrm{Gd}=\text { Gordura Digestível, } \mathrm{g} / \mathrm{kg}\end{array}$ & $\mathrm{G}=$ Gordura, $\% \quad \mathrm{~A}=$ Amido $\%$ \\
\hline $\mathrm{Gd}=$ Gordura Digestível, $\mathrm{g} / \mathrm{kg}$ & PB = Proteína, \% \\
\hline MOd = Matéria Orgânica Digestível, g/kg & $\mathrm{FB}=$ Fibra Bruta, $\%$ \\
\hline
\end{tabular}

๑) 2007 Sociedade Brasileira de Zootecnia 
Tabela 4 - Cálculo dos valores energéticos dos alimentos para aves e suínos usando equações (Rostagno et al., 2005).

\begin{tabular}{lccccc}
\hline \multirow{2}{*}{ Nutriente } & \multicolumn{2}{c}{ Milho } & & \multicolumn{2}{c}{ Farinha de Carne e Ossos } \\
\cline { 2 - 3 } \cline { 5 - 6 } & Tab. Bras. & Novo $^{\mathbf{1}}$ & & Tab. Bras. & Nova $^{\mathbf{2}}$ \\
\hline Proteína, \% & 8,26 & 7,26 & & 41,00 & 43,00 \\
Gordura, \% & 3,61 & 2,61 & & 11,04 & 13,04 \\
Fibra, \% & 1,73 & 2,73 & & - & - \\
EM aves, kcal/kg & 3379 & 3256 & & 1941 & 2109 \\
& & & & 2065 & 224 \\
EM suínos, $\mathrm{kcal} / \mathrm{kg}$ & 3390 & 3268 & & 1377 & 1506 \\
EL suínos, kcal/kg & 2681 & 2587 & & \\
\hline
\end{tabular}

1 - Redução de $1 \%$ da proteína e gordura e aumento de $1 \%$ da fibra. 2 - Aumento de $2 \%$ da proteína e gordura. Os outros nutrientes iguais aos das Tabelas Brasileiras.

A existência de instalações apropriadas contendo número suficiente de unidades experimentais (boxes, baias, gaiolas), que proporcionem condição ambiental uniformes, bem como a existência de máxima homogeneidade entre os animais, permite a escolha de um delineamento inteiramente casualizado. Em contrapartida, instalações físicas que não apresentem controle adequado das condições ambientais internas, animais com pesos iniciais ou genótipos diferentes, resultam em variações que se não forem devidamente controladas, podem alterar o efeito de significância do experimento. Neste caso, deve-se optar pela escolha do delineamento em blocos casualisados, separando os animais em blocos segundo o ambiente (ex. frio e calor), ou segundo o peso (ex. bloco de animais pesados e bloco de animais leves), o que resultará na redução da variação do experimento.

Atualmente, as novas tecnologias aplicadas na área da nutrição animal permitem ganhos adicionais do desempenho na ordem de 2 a $3 \%$. Embora pareçam ganhos pequenos, se considerarmos o número de milhares de animais existentes nas unidades produtivas, estes ganhos adquirem importância fundamental no âmbito econômico. No entanto, tais diferenças ao nível de pesquisa só podem ser detectadas se as variações existentes forem controladas ao máximo, garantido que as diferenças encontradas sejam realmente atribuídas ao efeito do tratamento.

Desta forma, é preciso que sejam definidas no planejamento, além das características avaliadas, a unidade experimental, o número de animais por unidade experimental e o número de repetições por tratamento, possibilitando que as variações entre as unidades experimentais (erro experimental), sejam as menores possíveis.
Para frangos de corte, suínos em crescimento e galinhas poedeiras a unidade experimental (UE) geralmente é boxe, baia ou gaiola, e o número de animais de cada UE irá depender da característica avaliada. No caso de frangos de corte, para avaliar características como ganho de peso, consumo de ração e conversão alimentar, a unidade experimental deve conter dezenas de frangos e, se as características avaliadas forem relacionadas á carcaça, como rendimento, percentagem de peito ou de coxas, o número de aves na unidade experimental pode ser reduzido uma vez que estas características não sofrem tanto o efeito do ambiente.

A uniformidade dos animais, com peso similar, mesmo sexo e mesma idade, é outro pré-requisito fundamental na formação das unidades experimentais. O objetivo é agrupar animais o mais uniformes possíveis, para que todos iniciem o experimento com as mesmas características. Pintos de 1 dia geralmente são mais uniformes e estão disponíveis em maiores quantidades, o que facilita a padronização na formação das UEs. Para animais cuja variação de peso é maior, como suínos e aves de postura, deve-se primeiramente uniformizar os pesos médios das parcelas e em seguida, realizar a distribuição por faixas de peso.

Adotando como exemplo um experimento composto de três tratamentos com oito repetições, totalizando 24 unidades experimentais de 20 pintos machos, cujo peso inicial possui alta variação (Tabela 5). Todos os pintos devem ser pesados com uma balança de divisão de uma grama e colocados em 9 caixas. Os pintos selecionados nas caixas 3 até 7, deverão ser distribuídos nas 24 unidades experimentais, resultando em pesos médios entre 41,15 a 41,65 gramas Tabela 6). Outra opção de distribuição das aves nas UEs seria distribuir os

() 2007 Sociedade Brasileira de Zootecnia 
Tabela 5 - Distribuição de pintos em caixas segundo o peso.

\begin{tabular}{lcccccccccc}
\hline Caixa N & $\mathbf{1}$ & $\mathbf{2}$ & $\mathbf{3}$ & $\mathbf{4}$ & $\mathbf{5}$ & $\mathbf{6}$ & $\mathbf{7}$ & $\mathbf{8}$ & $\mathbf{9}$ & Total \\
\hline Peso(g) & $<35$ & $36-37$ & $38-39$ & $40-41$ & $42-43$ & $44-45$ & $46-47$ & $48-49$ & $>50$ & \\
Pintos & 8 & 23 & 97 & 177 & 154 & 80 & 36 & 16 & 9 & 600 \\
1)Pintos & 0 & 0 & $72(3)$ & $168(7)$ & $144(6)$ & $72(3)$ & $24(1)$ & 0 & 0 & 480 \\
2)Pintos BL & 0 & 0 & $72(6)$ & $168(14)$ & 0 & 0 & 0 & 0 & 0 & 240 \\
2)Pintos BP & 0 & 0 & 0 & 0 & $144(12)$ & $72(6)$ & $24(2)$ & 0 & 0 & 240 \\
\hline
\end{tabular}

(x) número de aves por boxe. BL= Bloco Leve; BP= Bloco Pesado.Adaptado de Euclydes \& Rostagno (2001).

Tabela 6 - Médias de pesos de pintos para os tratamentos e repetições para o modelo inteiramente casualizado ou para o modelo de blocos casualizados.

\begin{tabular}{cccc}
\hline Repetição & Tratamento 1 & Tratamento 2 & Tratamento 3 \\
\hline 1 (B1.1) & $41,40(39,9)$ & $41,25(39,9)$ & $41,40(39,9)$ \\
2 (B1.2) & $41,15(39,9)$ & $41,60(39,9)$ & $41,65(39,9)$ \\
3 (B1.3) & $41,60(39,9)$ & $41,65(39,9)$ & $41,45(39,9)$ \\
4 (B1.4) & $41,35(39,9)$ & $41,40(39,9)$ & $41,45(39,9)$ \\
5 (B2.1) & $41,35(43,5)$ & $41,55(43,5)$ & $41,25(43.5)$ \\
6 (B2.2) & $41,20(43,5)$ & $41,30(43,5)$ & $41,45(43,5)$ \\
7 (B2.3) & $41,60(43,5)$ & $41,25(43,5)$ & $41,45(43,5)$ \\
8 (B2.4) & $41,25(43,5)$ & $41,25(43,5)$ & $41,25(43,5)$ \\
Média & 41,36 & 41,41 & 41,42 \\
\hline
\end{tabular}

Obs - Os pesos entre parênteses são simulados tendo como base os pesos apresentados na tabela 5. Adaptado de Euclydes \& Rostagno (2001).

pintos em dois blocos, leves (BL) e pesados (BP), e analisar o experimento como blocos casualizados.

A variação existente dentro das unidades experimentais, independentes do efeito dos tratamentos, é conhecida como erro experimental. O aumento do número de animais nas unidades experimentais, bem como no número de repetições, visa á diminuição do quadrado médio do resíduo (QMR), pela distribuição das possíveis variações existentes entre o maior número de animais, reduzindo assim o erro experimental. É necessário que o número de repetições seja suficiente para minimizar esta fonte de variação e para que as estimativas dos efeitos dos tratamentos sejam obtidas com a precisão necessária, para detecção de diferenças entre os mesmos, no nível de significância planejado.

Sakomura e Rostagno (2007) realizaram um estudo para avaliar o número de repetições por tratamento e número de animais por repetição em ensaios de desempenho com aves e suínos, publicados no período de 1997 a 2003. Experimentos com frangos de corte usaram em média 5 repetições de 27 aves, e experimentos com suínos em crescimento usaram em média 6 repetições de 4 animais por tratamento. Hoje em dia, para melhorar a precisão dos experimentos e detectar diferenças de 2 - 4\% entre os tratamentos, seria recomendável aumentar o número de repetições para 10 por tratamento com o mesmo número de animais citados acima.

Para estimar a variação experimental, uma medida utilizada pelos pesquisadores é o coeficiente de variação (CV), o qual representa o desvio padrão expresso como porcentagem da média $\mathrm{CV}=(\mathrm{s} /$ x)*100, onde s sé a raiz quadrada da variância residual, e $\underline{x}$ é a média geral do experimento. Este é um importante parâmetro de avaliação da precisão experimental e permite a comparação com outros ensaios (Sakomura \& Rostagno, 2007). Na Tabela 7 estão os coeficientes de variação relatados de experimentos com frangos de corte, galinhas poedeiras e suínos.

Um cuidado adicional que merece destaque é a formulação correta das rações experimentais, com atenção especial à mistura dos ingredientes. A avaliação de diferentes alimentos ou de níveis de um nutriente, quando as rações não são adequadamente misturadas, compromete definitivamente os resultados. Uma sugestão para uma mistura mais precisa seria o método da diluição, onde a partir de rações (basais) contendo os níveis extremos do alimento ou do nutriente avaliado (nível mais alto e nível mais baixo) se obtém rações com níveis intermediários.

\section{Processamento dos dados experimentais}

Os dados obtidos nos ensaios de produção 
Tabela 7 - Faixa tolerável de coeficiente de variação (CV) em diferentes parâmetros.

\begin{tabular}{|c|c|c|c|}
\hline \multirow[b]{2}{*}{ Variável } & \multicolumn{3}{|c|}{ Faixa de Coeficiente de Variação (CV, \%) } \\
\hline & Baixo & Médio & Alto \\
\hline \multicolumn{4}{|l|}{ Frangos de Corte ${ }^{1}$} \\
\hline Ganho de peso & $<1,4 \%$ & $2,9 \%$ & $>7,1 \%$ \\
\hline Conversão alimentar & $<0,8 \%$ & $2,4 \%$ & $>4,6 \%$ \\
\hline \multicolumn{4}{|l|}{ Poedeiras $^{1}$} \\
\hline Produção de Ovos & $<3,1 \%$ & $6,7 \%$ & $>13,2 \%$ \\
\hline Peso de Ovos & $<1,2 \%$ & $4,4 \%$ & $>20,0 \%$ \\
\hline \multicolumn{4}{|l|}{ Suínos ${ }^{2}$} \\
\hline Ganho de peso & $<6,1 \%$ & $6,1-17,8 \%$ & $>17,8 \%$ \\
\hline Rendimento de carcaça & $<1,2 \%$ & $1,2-3,1 \%$ & $>3,1 \%$ \\
\hline
\end{tabular}

1 - Faixas fornecidas por Sakomura \& Rostagno (2007). 2 - Faixas fornecidas por Judice (1999).

planejados e conduzidos adequadamente, devem ser submetidos á análises estatísticas. A escolha do teste estatístico deve ser pré-estabelecida, ainda na fase de planejamento, e exige conhecimentos de estatística e da espécie animal avaliada.

$\mathrm{Na}$ atualidade o nível de significância não precisa ser fixado somente em $5 \%$ podendo variar para 7 ou $10 \%$, dependendo da importância econômica da variável estudada (ganho de peso, conversão alimentar, rendimento de carcaça, etc.).

A análise de variância (ANOVA) é o primeiro passo na análise dos resultados obtidos nos ensaios de crescimento, permitindo testar a hipótese de igualdade das médias dos tratamentos pelo teste $\mathrm{F}$.

Para determinar o melhor nível do nutriente estudado, isto é, para parâmetros quantitativos, na avaliação de alimentos e também das exigências nutricionais, o uso da análise de regressão é o mais apropriado, onde uma função é construída entre parâmetro de resposta (Y) e os fatores em estudo (X). Parâmetros de ordem qualitativa (ex. milho vs sorgo), no entanto, devem ser analisados apenas com aplicação de testes de médias. O teste de média recomendado para experimentos de desempenho com animais monogástricos é o de StudentNewman-Keul's. Entretanto outros testes podem ser aplicados de acordo com os objetivos do experimento e os parâmetros avaliados.

\section{Exigências nutricionais - regressão}

Para estimar as exigências nutricionais das aves e dos suínos são realizados experimentos de desempenho, também chamados de experimentos dose-resposta. $\mathrm{O}$ método dose-resposta estima a exigência nutricional de determinado nutriente pela avaliação de uma resposta a parâmetros prédefinidos, como ganho de peso, conversão alimentar, deposição de carne magra, produção de ovos no período, através do oferecimento de quantidades crescentes do nutriente (níveis) nas dietas. Neste método, a exigência do animal de um nutriente se dá no nível máximo de resposta nos parâmetros avaliados, como máximo ganho de peso, por exemplo. Os resultados (exigência) podem ser diferentes segundo o critério de resposta ou modelos de regressão utilizados (modelo descontínuo - Linear Response Plateau (LRP), modelo quadrático e modelo exponencial) para estimar o nível ótimo do nutriente.

Euclydes \& Rostagno (2001) citam que a aplicação de cada um dos modelos dependerá da relação entre os níveis do nutriente em estudo e a resposta aos mesmos, podendo haver subestimação do nível ótimo, no caso do LRP. Já a função quadrática que aparentemente apresenta vantagem na determinação da exigência nutricional por estimar o desempenho máximo possível, apresenta desvantagens, no sentido de ser muito sensível ás diferenças entre os níveis estudados, além de apresentar simetria bilateral, ou seja, descreve a queda na produção na mesma intensidade do acréscimo entre os níveis, o que biologicamente parece não ser adequado (Figura 1).

A combinação dos dois modelos seria a melhor recomendação, segundo Euclydes \& Rostagno (2001), mediante o uso de equação quadrática de resposta, associada ao platô. A vantagem deste modelo é que o nível ótimo encontrado não é alto como o geralmente estimado pela derivação da função quadrática, nem tão baixo como o observado no modelo LRP, passando a ser intermediário, no ponto de encontro entre a reta da equação e o platô. Outra opção usada pelos pesquisadores seria a aplicação de um intervalo de confiança de

๑ 2007 Sociedade Brasileira de Zootecnia 
95\% do valor estimado pela equação quadrática, no caso da figura 1 então, a exigência estimada seria: $0,79 \times 95 / 100=0,75 \%$ de lisina.

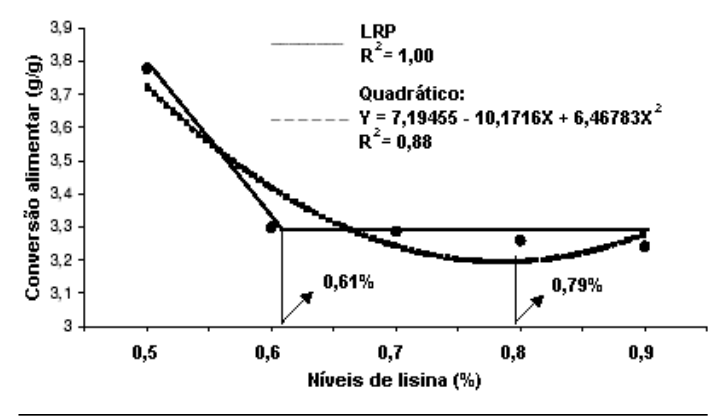

Figura 1 - Estimativa da exigência nutricional de lisina obtida mediante o modelo LRP e Quadrático para ótima conversão alimentar de suínos machos castrados dos 96 aos 126 kg (Arouca et al., 2003).

Para comparar as metodologias usadas para estimar as exigências nutricionais (LRP, quadrática, 95\% da quadrática, quadrática com platô e exponencial), Euclydes \& Rostagno (2001) usaram os dados de duas teses de doutorado do Programa de Pós-graduação em Zootecnia da Universidade Federal de Viçosa. Os autores Barboza (1998) e Costa (2000), utilizaram frangos de corte, macho, provenientes das empresas Hubbard (2 experimentos) e Ross (2 experimentos). O nível energético das rações foi de $3200 \mathrm{Kcal} / \mathrm{Kg}$ e os períodos experimentais foram de 15-40 dias (2 experimentos) e 22-40 dias (2 experimentos). As características analisadas foram: ganho de peso $(\mathrm{g})$, conversão alimentar e peito com osso (\%). As análises de regressão foram executadas com as médias das características para cada nível de lisina digestível, como apresentado na Tabela 8. Com a aplicação de todas as metodologias propostas, a percentagem média de lisina digestível recomendada na formulação de rações para frangos de corte, machos, no período de 15/22 - 40 dias de idade, apresentou pouca variação: 0,948, 0,947 e $0,963 \%$, para ganho de peso, conversão alimentar e peito com osso, respectivamente.

\section{Exigências nutricionais - método fatorial}

$\mathrm{O}$ método fatorial estima as exigências nutricionais pelo cálculo das demandas por nutrientes para as principais funções metabólicas do animal, divididas em funções de mantença e crescimento. Este método tem sido apresentado sob a forma de vários modelos matemáticos, que permitem estimar as exigências em nutrientes, considerando características como diferenças genéticas, peso corporal ou fase (pré-inicial, inicial, crescimento e terminação), nível de rendimento ou a capacidade de deposição de carne, aspectos relacionados ao ambiente e seus efeitos sobre os animais, e ainda, informações do potencial nutritivo dos alimentos e o efeito da complementaridade entre eles (Pomar \& Dit Bailleul, 1999).

As predições destas exigências se baseiam em dados experimentais obtidos com ensaios de produção (dose resposta), levando em consideração ainda, a eficiência de utilização metabólica dos nutrientes para o crescimento e mantença.

Nas Tabelas Brasileiras (Rostagno et al., 2005) é usado o modelo fatorial para estimar as exigências nutricionais de aves e suínos. Segundo

Tabela 8 - Ganho de peso, conversão alimentar e peito com osso, de frangos de corte machos e exigências de lisina digestível, estimadas com diferentes metodologias.

\begin{tabular}{cccc}
\hline Lisina Digestível (\%) & Ganho de Peso & Conversão & Peito com osso \\
\hline 0.772 & 1362.9 & 1.94 & 28.58 \\
0.832 & 1428.9 & 1.87 & 29.43 \\
0.892 & 1500.1 & 1.81 & 29.96 \\
0.952 & 1525.3 & 1.77 & 30.57 \\
1.012 & 1528.9 & 1.78 & 30.51 \\
1.072 & 1507.0 & 1.78 & 30.47 \\
\hline Métodos Aplicados & Nível de Lisina digestível recomendado (\%) \\
\hline LRP “Linear Response Plateau” & 0,915 & 0,915 & 0,935 \\
Equação quadrática com platô & 0,910 & 0,916 & 0,943 \\
Equação quadrática & 0,992 & 1,002 & 1,015 \\
Eq. quadrática 95\% do Max. & 0,942 & 0,952 & 0,964 \\
Exponencial + Bom senso & 0,980 & 0,950 & 0.960 \\
\hline
\end{tabular}

Euclydes \& Rostagno (2001).

๑ 2007 Sociedade Brasileira de Zootecnia 
os autores, este modelo permite flexibilizar as exigências, pois desta maneira pode-se calcular o nível nutricional adequado de cada animal de acordo com o desempenho esperado, evitando desta maneira excessos que aumentam a contaminação ambiental, ou deficiências que reduzam a produtividade.

Na Tabela 9 é apresentada a equação usada para calcular a exigência de lisina em g,/dia de suínos machos castrados com diferentes desempenhos (regular, médio e superior), a porcentagem de lisina é então calculado levando em consideração o consumo diário de ração. Para estimar as necessidades dos outros aminoácidos os autores utilizaram o conceito de Proteína Ideal. variar conforme a importância econômica da característica estudada.

A adequada condução dos ensaios, sejam de crescimento ou de digestibilidade, é fundamental para que as ferramentas apresentadas possam ter efetividade, resultando na melhora da produtividade e na redução dos custos e da excreção de nutrientes.

\section{Literatura citada}

ABREU, M.L.T; DONZELE, J.L.; SUIDA, D. et al. In: I Congresso Latino Americano de Suinocultura - Foz do Iguaçu. p. 200-217. 2002.

AROUCA, C.L.C.; FONTES, D.O.; VELOSO, J.A.F et al. Níveis de lisina para suínos machos castrados selecionados

Tabela 9 - Exigência de lisina digestível de suínos, machos castrados, de diferentes desempenhos calculada com a equação de Rostagno et al. (2005).

\begin{tabular}{lccc}
\hline \multicolumn{4}{c}{ Lis. Dig. $(\mathrm{g} / \mathrm{dia})=0,036 \mathrm{P}^{0,75}+\left(11,467+0,2505 \mathrm{P}-0,0016 \mathrm{P}^{2}\right) \mathrm{G}$} \\
& $\mathrm{P}=$ Peso Corporal Médio em kg; $\mathrm{G}=\mathrm{Ganho} / \mathrm{dia}$ em kg \\
\hline Desempenho & Regular & Médio & Superior \\
Peso Médio, kg & $\mathbf{6 0}$ & $\mathbf{6 0}$ & $\mathbf{6 0}$ \\
\hline Ganho de Peso, kg/dia & 0,862 & 0,960 & 1,080 \\
Consumo, kg/dia & 2,680 & 2,494 & 2,430 \\
Exig. Lis. Dig., g/dia & 18,793 & 20,711 & 23,172 \\
Lis. Dig. na Ração, \% & 0,696 & 0,829 & 0,953 \\
\hline
\end{tabular}

\section{Conclusões}

O avanço no conhecimento na área de avaliação dos alimentos e das exigências nutricionais de animais monogástricos se dá a passos largos, devido á seriedade com que os profissionais encaram a responsabilidade de fazer pesquisa de qualidade dentro e fora do país.

Neste trabalho foram abordadas as metodologias que permitem melhorar a utilização dos alimentos de maneira mais eficiente e econômica. Foram também citados os cuidados e procedimentos essenciais para executar adequadamente experimentos de desempenho com aves e suínos.

$\mathrm{Na}$ atualidade, para a realização de experimentos com monogástricos, é necessário: definir claramente os objetivos, utilizar animais com peso inicial uniforme, usar número adequado de repetições e de animais por unidade experimental.

Testes de médias devem ser usados para as variáveis qualitativas e quando a variável independente for quantitativa aplicar análise de regressão. O nível de significância utilizado pode geneticamente para alta eficiência de crescimento, dos 96 a0s $120 \mathrm{~kg}$ I - Desempenho. In: XI CONGRESSO BRASILEIRO DE VETERINÁRIOS ESPECIA-LISTAS EM SUÍNOS, Goiânia, Anais...Goiânia, 2003, p. 259-260.

BAILLEUL, J.D.P.; BERNIER, J.J.; POMAR, C. Journal Rech. Porcine France, v.30, p:127-132, 1998.

BARBOSA, R.J. Exigência de Metionina-Cistina para frangos de corte na fase de crescimento a acabamento. Tese (Mestrado em Zootecnia) - Universidade Federal de Viçosa, Viçosa. 82 p.,1998.

BÜNZEN, S.; ROSTAGNO, H.S.; LOPES, D.C. et al. Digestibilidade verdadeira do fósforo de alimentos determinada com suínos em crescimento e terminação. Congresso Latino-Americano de Nutrição Animal - CLANA, 2006. São Paulo, SP.

CVB. Veevoedertabel: Gegevens over chemische samenstelling, verteerbaarheid envoederwaarde van voedermiddelen). Central Veevoeder Bureau, Lelystad, Países Bajos. 1998.

EUCLYDES, R.F.; ROSTAGNO, H.S. et al. Estimativa dos níveis nutricionais via experimentos de desempenho. In: IWORKSHOP LATINO-AMERICANOAJINOMOTOBIOLATINA, 2001, FoZ do Iguaçu, Anais... Foz do Iguaçu. p.77-88

JACKSON, D.A.; DALIBARD, P. et al. The measurement and validation of the technique of using digestible amino acids for diet formulation in poultry and swine. In: SIMPÓSIO LATINO AMERICANO DE NUTRIÇÃO DE SUÍNOS E AVES, Anais... Campinas, SP, 47-72, 1995.

JUDICE, M.G.; MUNIZ, J.A.; CARVALHEIRO, R. et al. Avaliação do coeficiente de variação na experimentação com suínos. Ciência e Agrotecnologia, v.23, n.1, p.170-173, 1999.

NOBLET J.; FORTUNE, H.; SH., X.S. et al. Prediction of net energy value of feeds for growing pigs. J. Anim. Sci., v.72, p. 344-354, 1994b.

๑ 2007 Sociedade Brasileira de Zootecnia 
NOBLET, J.; SEVE, B.; TRAN, G. et al. Valoración energética y protéica de alimentos para porcino: Propuestas Francesas. XX CURSON DE ESPECIALIUZACIÓN AVANCES EN NUTRICIÓN Y ALIMENTACIÓN ANIMAL. FEDNA. España:Fundación Española para el Desarrollo de la Nutrición Animal. Barcelona, 2004.

POMAR, C.; BAILLEUL, D.P.J. et al. Determinación de las necessidades nutricionales de los cerdos de engorde: Limites de los métodos actuales. XV CURSON DE ESPECIALIZACIÓN AVANCES EN NUTRICIÓN Y ALIMENTACIÓN ANIMAL. Madrid, España:Fundación Española para el Desarrollo de la Nutrición Animal. 1999. 301 pp. 253-276.

PUPA, J.M.R.; LEÃO, M.J.; CARVALHO, L.G. et al. Cecectomia em galos sob anestesia local e incisão abdominal.
Arq. Bras. Med. Vet. Zootec., v.50, n.5, p. 531-535, 1998 ROSTAGNO, H.S.; ALBINO, L.F.T.; DONZELE, J.L. et al. Tabelas brasileiras para aves e suínos. Viçosa, MG: UFV, 186 p. 2005.

ROSTAGNO, H.S.; PUPA, J.M.R.; PACK, M. Diet formulation for broilers base don total versus digestible amino acids. $\mathbf{J}$. Appl. Poult. Res., v.4, p.293-299, 1995.

SAKOMURA, N.K.; ROSTAGNO, H.S. Métodos para avaliação biológica dos alimentos e exigências nutricionais para animais monogástricos. Jaboticabal, SP, UNESP, (no prelo). 2007.

VAN MILGEN, J.; NOBLET, J. Energy partitioning in growing pigs: the use of a multivariate model as an alternative for the factorial analysis. J. Anim. Sci., v.77, p.2154-2162,1999. 